\title{
A process model for forecasting conditions conducive to blow-up fire events
}

\author{
$\underline{\text { R.H.D. McRae }}^{\text {a }}$ and J.J Sharples ${ }^{b}$ \\ ${ }^{a}$ ACT Emergency Services Agency, PO Box 158, Canberra, ACT 2605, Australia \\ Email: rick.mcrae@act.gov.au \\ ${ }^{b}$ Applied and Industrial Mathematics Research Group, School of Physical, Environmental and Mathematical \\ Sciences, University of New South Wales, Canberra, ACT 2600,
}

\begin{abstract}
Extreme fires are responsible for devastating losses in recent years in Victoria, New South Wales and the ACT. Detailed case studies have revealed that an extreme fire will include one or more blowup fire events. The importance of this is that, unlike extreme fires, blow-up fire events are relatively predictable. Using the outcomes from a number of recent studies, we believe that it is becoming feasible to forecast conditions conducive to blow-up fire events.
\end{abstract}

Here we present a process model for the purposes of forecasting conditions conducive to blow-up fire events.

Implementing the model requires collaboration between the fire agency duty officer and the duty forecaster. They require information about: the fire and from the fire ground; forecasts and observations; and specific data on terrain. This collaboration needs to be more formal than current arrangements, as no one participant can be expected to have sufficient information on-hand to produce a forecast of a blow-up fire event.

This information is used to answer a series of questions, arranged as steps in a flowchart, which will either loop-back or lead to the inference of conditions conducive to blow-up fire conditions. The steps in the flowchart have differing timeframes associated with them, firstly for impact lead time ahead of blow-up event formation, and secondly for predictability. Predictability of weather events includes both forecasting their occurrence and tracking their approach or development. These elements used by the model need to occur together, which may happen at different times in different places, due to the differing dynamics of the weather and the fire.

A key element of the model is separating elements of existing fire weather indices into components. Two key examples are discussed. The Fire Danger Index is equivalent to wind speed divided by fuel moisture content (FMC). FMC in turn reflects the difference between temperature and relative humidity. The Continuous Haines Index is based on a lapse rate term and a dryness term. While each index is useful, we demonstrate that working with their components provides greater functionality.

In addition, the movement of a fire into fire channelling prone terrain, may reflect suppression operations, which could prevent a blow-up event forming or, in a worst-case scenario, cause one.

The utility of the model is demonstrated in the context of a number of recent notable fires.

Keywords: Fire weather forecasting, extreme fires, blow-up fire event 
McRae and Sharples, A process model for forecasting conditions conducive to blow-up fire events.

\section{INTRODUCTION}

The wildfire industry has long focused primarily on surface weather forecasts, a minor element of the output of weather prediction systems, along with fuel loads and simple terrain metrics. In recent years there has been a growing awareness of the importance of the vertical structure of the lower atmosphere in the formation of extreme wildfires (Fromm et al., 2010).

The Haines Index (Haines, 1988) was the prime result of this, augmented in recent years by the Continuous Haines Index (Mills \& McCaw, 2010). However there was only a limited ability to link the surface and profile approaches into a single forecasting continuum (Bally, 1995). The Haines approach is a combination of lapse rate and dew point depression, while the surface-oriented fire danger measures are a mix of wind speed and fuel dryness (Sharples, et al., 2009).

At the same time that this was evolving, a series of case studies into extreme fires were highlighting their common elements, in terms of both the precursors and the blow-up mechanism (Fromm et al., 2006, Fromm et al., 2010, Fromm et al., 2012). A blow-up fire event may be defined as a discrete coupled fire-atmosphere event, characterized by uniformly severe intensity, well-defined extent and duration of two to three hours (McRae \& Sharples, 2011). The use of a wide range of observational technologies in these case studies has thrown new light onto the forecasts and the standard indices that are in operational use.

It is now clear that an extreme fire will comprise one or more blow-up fire events. The total fire is a mix of: blow-up fire events; fire spread under less severe conditions; containment efforts; and suppression efforts. This mix is still problematic to predict in detail. However, blow-up fire events are far more amenable to prediction. Historically, blow-up fire events cause the majority of fire impacts, and therefore attempting to forecast them will be a useful advance. It is the goal of this paper to present a process model for the purpose of forecasting conditions conducive to blow-up fire events. It must be noted that details of its development, the basis for selection of the questions used and proposed implementation are outside the scope of this paper.

\section{SETTING}

The fundamental basis of weather forecasting is the use of Numerical Weather Prediction (NWP) systems. These are models that simulate all or part of the atmosphere, and which work within a formal data structure. They predict a future state by applying physical rules to the current state. The models include three spatial dimensions and time, and work with a range of weather parameters.

Blow-up fire events arise from a combination of the fire's state (its extent, combustion dynamics and convective dynamics), the terrain on which the fire is burning and a range of weather events. Only the latter may be resolved in a NWP forecast run, necessitating a different approach to this forecasting challenge. To adequately describe the fire's current and future setting the data sources are the fire agency (for field observations of the fire and its setting), a web site (www.highfirerisk.com.au) (for terrain data) and the weather service (for weather observations, forecasts and assessments). Implementation of the process model under discussion requires an obligatory collaboration between the fire agency and the weather service.

The forecaster typically works across the entire operational domain but the fire agency works on a fire-byfire basis. For this reason the initial focus of blow-up fire event forecasting needs to be restricted to an ongoing fire. Perhaps in future, after skill accumulation, this might expand to cover potential fires.

The role of the earth's surface in a NWP includes shaping the data structure, absorption of solar radiation and providing surface roughness for estimation of turbulence. In contrast a fire will have a landform setting within which it is burning, and will burn onto in the immediate future. Traditionally terrain is described for fire purposes by elevation (for adiabatic lapse rate effects on temperature), slope (for burning efficiency) and aspect (for alignment with the wind). For this model we need additional data. Terrain ruggedness for a point is defined as the range of elevation present within a $1500 \mathrm{~m}$ radius. If this range exceeds $300 \mathrm{~m}$ it is classed as rugged, if it is under $150 \mathrm{~m}$ it is classed as flat, otherwise it is classed as undulating. The other terrain descriptor, fire channelling prone lands, refers to steep slopes that are nearly perpendicular to the prevailing wind (Sharples, et al., 2012).

The fire will also have a weather setting. This will mostly be the result of the continuous diurnal cycles of weather, driven by variations in solar energy input. Superimposed on this will be gross changes due to the advection of a different air mass and localized discrete weather events, most of which involve the vertical flow of air. Much of this involves interactions with the terrain, making the depiction of the terrain within the NWP system of critical importance. Currently operational weather models, with a horizontal resolution of $\sim 5 \mathrm{~km}$, are too coarse to resolve a fire's landform setting. Post-input from duty forecasters to account for this resolution mismatch, using fire-ground information, therefore remains essential. 
At present, if the blow-up fire event forecasting model was to be implemented the data sources would be as follows.

Fire descriptions from the fire ground would need to be of sufficient detail to fully relate the weather and terrain settings. We believe that a resolution of broadly kilometer-scale would suffice.

The terrain data for Australia, indicating ruggedness and fire channelling prone lands, are being provided online at the HighFire Risk Project's website. This currently covers southeast New South Wales, the ACT, Victoria, Tasmania and southwest Western Australia (see Figure 1). It will be extended as resources permit.

The weather data are from the Australian Digital Forecast Database, a data system used by the Bureau of Meteorology for managing its NWP output products. Special Fire Weather Forecasts are prepared by the Duty Forecaster, based on the ADFD data and on field data from the fire agencies. It should be noted that while this paper refers to the Australian Bureau of Meteorology, all concepts are applicable in other countries.

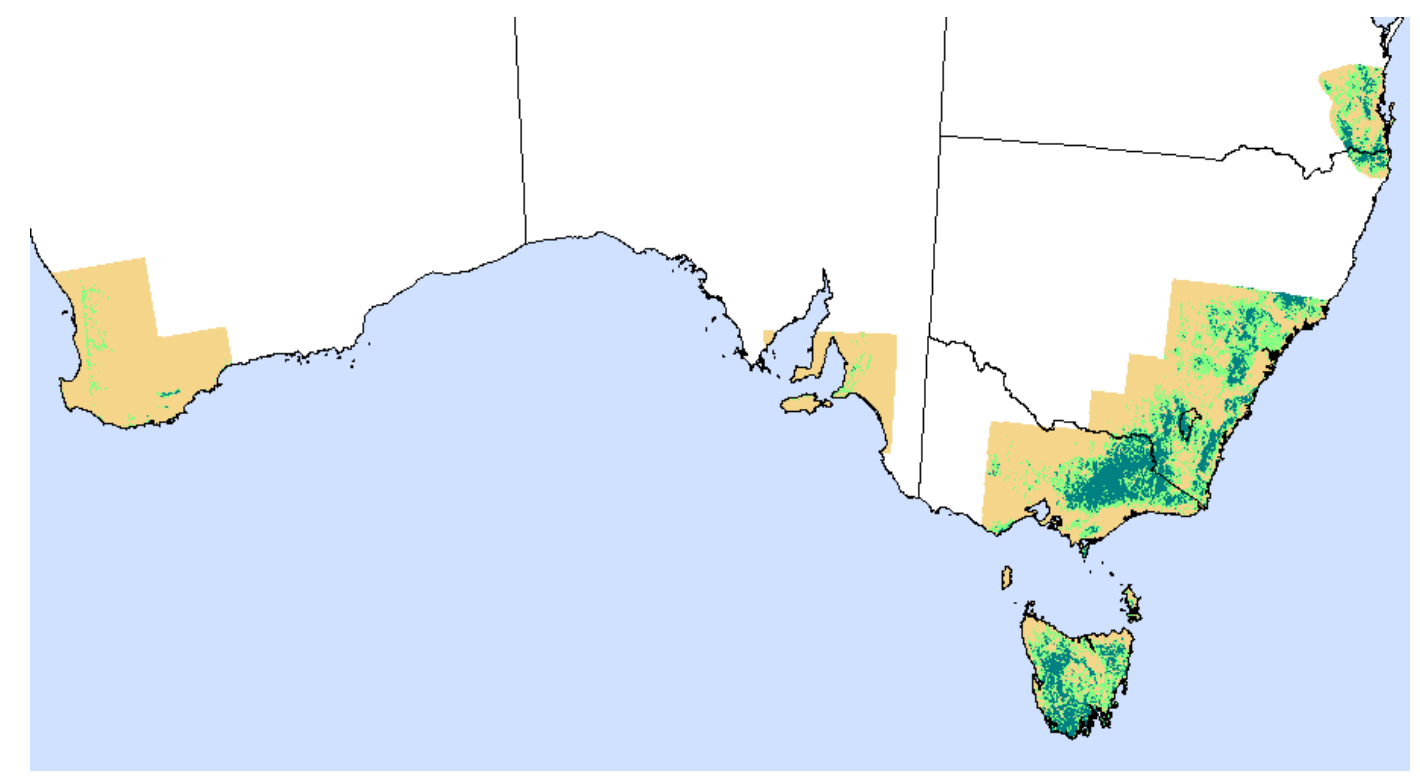

Figure 1. Progress-to-date on terrain classification for Australia. (Beige indicates flat terrain, light green indicates undulating terrain, and dark green indicates rugged terrain).

\section{PROCESS MODEL}

The process model (Figure 2) is based on a path leading from a clearly defined start point and a series of questions (conditional clauses), based on the fire and it's setting. Each question is formulated to provide a path dichotomy, with the "yes" option leading to further questions or to inferred occurrences of interest. The "no" option is used to loop back to allow re-running of the model at a future time (either to a schedule or when new information becomes available). The ultimate, inferred occurrence is that of a blow-up fire event, and from there the model may be re-run at a future time. This loop-back is essential as some fire complexes may repeatedly blow-up. Examples of this include the 2003 and 2006 Victorian Alpine Complex and the West Fork (Colorado) Complex of July 2013 (Mike Fromm, pers. comm.). Three other inferred occurrences might be derived as intermediate, but nevertheless potentially useful, model outputs. The model also formally ceases when there is no uncontrolled fire present.

There are four separate conditions that are used to infer dry fuel - a dry slot (Mills, 2005); a low dew point event (Sharples, 2009); a foehn wind (Sharples, et al. 2009b); and a large difference in the values of temperature and relative humidity (Sharples, et al., 2009a) - and these are checked separately as needed by the model dynamics.

Each question in the model refers to a condition (for example winds in excess of a threshold value) being forecast (or already occurring). If it is, then the model provides some guidance as to four properties of the forecast condition. These are: (i) the forecast lead-time before impact; (ii) the lead-time over which the event may be tracked as it approaches; (iii) the time between occurrence and impact on the fire's behavior; and (iv) the time taken to verify the occurrence. These times are given in hours. If used correctly these timings could be valuable in developing community advices and warnings. 
Two of the key tools in use for fire management in Australia at present are the Fire Danger Index (FDI) and the Continuous Haines Index (CHI). It has been demonstrated that both indices may be split into two useful components (Sharples et al. 2009 and Mills \& McCaw, 2010).

FDI reflects wind speed divided by fuel moisture content (FMC). It is important to note that a single elevated value of FDI may reflect strong winds, low FMC or some combination of both. Quite different fire behavior would result from each possibility.

$\mathrm{CHI}$ reflects the dew point depression at the $850 \mathrm{hPa}$ pressure level and lapse rate between that level and the $700 \mathrm{hPa}$ level. The dew point term indicates the availability of dry air to be mixed downwards and produce a low FMC event, and the lapse rate term, which if manifested as an instability band reflects the ability for a fire plume to rise unimpeded, producing different potential fire effects. Again, any given value of CHI may derive from varying combinations of its components, with differing effects on wildfire dynamics.

A significant proportion of blow-up fire events occur at night. This is counter to the usual expectation of FDI following a diurnal cycle and peaking in early to mid-afternoon. This is why FDI has no explicit role in the model (it is only a boundary condition for application of the model). Other events occur earlier in the day than might be expected. Examination of the process model shows a desirable reliance on factors that are not necessarily tied to the typical diurnal weather cycle. Winds may be elevated at any time, especially in response to the approach of air mass changes. Terrain clearly is independent of weather. Also in use is the difference between the values of temperature and relative humidity. That difference may peak at night in response to subsidence inversions, low-level jets or foehn winds. An active fire may mix down drier upper air at any time.

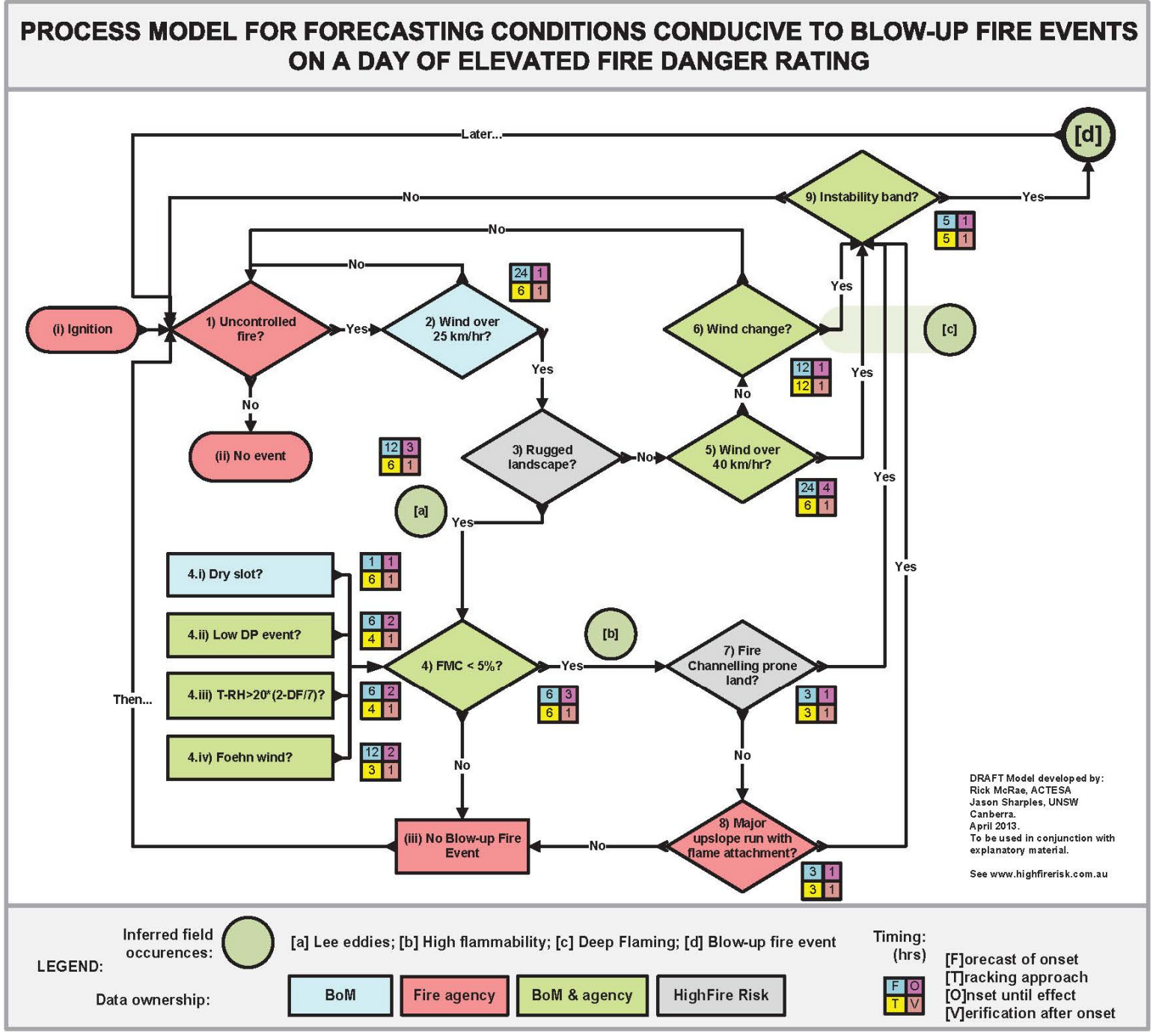

Figure 2. The process model. 
McRae and Sharples, A process model for forecasting conditions conducive to blow-up fire events.

In the traditional forecasting approach, the dynamics of a wildfire at any time may be estimated from the forecast state of its environment. If the environmental elements vary continuously, then the fire dynamics will also vary continuously. The impact of intervention efforts such as suppression may be factored in through alteration to the spatial extent of the fire.

What we are describing here is a system that includes a set of conditional clauses as proxies for the spatiotemporal coincidence of a critical set of environmental elements. As an example, if the fire arrives at a given point when the band of instability passes overhead, then its dynamics will be totally different compared to if the two arrive some hours apart. The environmental elements may not vary continuously, and the fire dynamics may subsequently undergo a phase change and vary discontinuously. This discontinuity is what is called a blow-up fire event. The environmental discontinuities are the subject of some of the conditional clauses.

One surprising implication from the model is that an attempt by a fire service to hold a fire at a containment option that ultimately fails may be the cause of the fire arriving subsequently at a place and time where a blow-up fire event can develop. Had the attempt not occurred, such as by selecting more distant control lines, the fire may have passed through that place at an earlier time without all requirements for a blow-up fire event being in place.

\section{EXAMPLES}

Ti-Tree Creek Fire of December 17, 2009 (near Michelago, NSW):

A fire started on a day of Catastrophic Fire Danger Rating. The FDR peaked in the early afternoon, and this peak occurred on a deepening trough-line. The outbreak was initially on undulating, fully cured grassland. Due to the FDR, suppression efforts failed. Just over an hour later the fires entered rugged terrain on the Tinderry Mountains. Shortly afterwards fire channelling occurred and a blow-up fire event followed.

Application of the model to this scenario would rapidly agree to questions 1 and 2 in Figure 2. Winds were not over $40 \mathrm{~km} / \mathrm{hr}$, and the model would thus loop back. The next task would be using fire behavior analyses to estimate the time to entry onto the rugged landscape. This would be of the order of 1 hour. So the next hour the model would rapidly agree to questions $1,2,3,4,7 \& 8$ and at that point infer the occurrence of a blow-up fire event. Therefore one hour after the outbreak of fire a blow-up fire event might occur. The preparation and issuing of specific public warnings would be a challenge, needing to be initiated immediately. As expected at the time, a blow-up fire event did occur. A stylized threat footprint was used operationally.

McIntyres Hut, Bendora \& Broken Cart Fires and Stockyard Complex (ACT \& NSW), January 18, 2003:

On the $8^{\text {th }}$ of January 2003, lightning in the ranges west and southwest of Canberra started a number of wildfires. For ten days these burnt as somewhat predictable but problematic fires. Typically flowchart questions 1, 2 \& 3 were true, but question 4 (low FMC) was false, forcing a loop-back (see Figure 2).

As described in Mills (2005), on the $18^{\text {th }}$ conditions deteriorated dramatically. Question 4 went to true. At this stage the fires were on a number of sites in the rugged landscape that were supportive of the development of fire channelling under the prevailing winds (Sharples et al., 2012). Mills (2005) showed that the fires formed a set of pyrocumulonimbi as a dry slot crossed each fire area. Thus questions $7 \& 9$ became true and a series of blow-up fire events could be inferred. Mills also showed how water vapor imagery permitted tracking of the approach of instability through its atmospheric manifestations. This indicates how the model may provide enough lead-time to issue warnings in such circumstances.

\section{CONCLUSIONS}

This paper shows that it is feasible to use a process model for the prediction of the potential for blow-up fire events to occur. Given that these events are the most damaging type of fire there is significant benefit to the community if any refinement to existing forecasting skills can be made.

Established methods treat the fire as a surface process that reflects surface weather, terrain and fuel. Recent research has made it clear that vertical airflow is frequently involved in the production of a coupled fireatmosphere event, which will typically become a blow-up fire event.

The complexities of combining surface and vertical parameters for this process model dictated its current morphology. This structure provided an ability to include timing, including anticipation of the arrival at the fire of key meteorological events. It also permits the inference of important intermediate processes. 
McRae and Sharples, A process model for forecasting conditions conducive to blow-up fire events.

The collaboration required to implement this model raises issues to do with ownership of the process. Recent enquiries and litigation arising from bushfires necessitates consideration of liability that may arise from the model's use. These issues are yet to be addressed, let alone resolved.

As our understanding of blow-up fire events improves, it is anticipated that this model will evolve in response. The model will be further refined through testing on days of elevated fire danger, through working with forecasters and fire agencies and through the findings of future case studies.

\section{REFERENCES}

Bally, J. (1995). The Haines Index as a predictor of fire activity in Tasmania. Proceedings, 1995 Fire Weather Workshop, Hahndorf, South Australia.

Fromm, M., Lindsey, D.T., Servranckx, R., Yue, G., Trickl,, T., Sica, R., Doucet, P. \& Godin-Beekmann, S. (2010). The untold story of pyrocumulonimbus. Bulletin of the American Meteorological Society. September 2010, 1193 - 1209.

Fromm, M.D., McRae, R.H.D., Sharples, J.J. \& Kablick, G.P (2012). Pyrocumulonimbus pair in Wollemi and Blue Mountains National Parks, 22 November 2006. Australian Meteorological and Oceanographic Journal, 62, 117-126.

Fromm. M, Tupper, A, Rosenfeld, D, Servrancx, R \& McRae, R, (2006). Violent pyro-convective storm devastates Australia's capital and pollutes the stratosphere. Geophysical Research Letters 33, L05815.

Haines, D.A. (1988). A lower atmosphere severity index for wildland fire. National Weather Digest, 13, 2327.

McRae, R. \& Sharples, J. (2011). A conceptual model for assessing the risk posed by extreme bushfires. Australian Journal of Emergency Management, 26(2), 47-53.

Mills, G.A. (2005). On the sub-synoptic scale meteorology of two extreme fire weather days during the Eastern Australian fires of January 2003. Australian Meteorological Magazine, 54, 265-290.

Mills, G.A. \& McCaw, L. (2010). Atmospheric Stability Environments and Fire Weather in Australia extending the Haines Index. CAWCR Technical Report No. 20.

Sharples, J.J. (2009). An overview of mountain meteorological effects relevant to fire behaviour and bushfire risk. International Journal of Wildland Fire 18, 737-754 doi:10.1071/WF08041.

Sharples, J.J., McRae, R.H.D., Weber, R.O. \& Gill, A.M. (2009a). A simple index for assessing fire danger rating. Environmental Modelling and Software 24, 764-774.

Sharples, J.J., McRae, R.H.D. \& Wilkes, S.R. (2012). Wind-terrain effects on the propagation of wildfires in rugged terrain: fire channelling. International Journal of Wildland Fire, 21, 282-296.

Sharples, J.J., Mills, G.A., McRae, R.H.D., \& Weber, R.O. (2009b). Fire danger anomalies associated with foehn-like winds in southeastern Australia. 18th World IMACS/MODSIM Congress, Cairns. 